\title{
The scanner as a stressor: Evidence from subjective and neuroendocrine stress parameters in the time course of a functional magnetic resonance imaging session
}

\author{
Markus Muehlhan ${ }^{\text {a,b,c, }}$, Ulrike Lueken ${ }^{\text {b,c }}$, Hans-Ulrich Wittchen ${ }^{b}$, Clemens Kirschbaum ${ }^{\text {a }}$ \\ a Chair of Biopsychology, Department of Psychology, Technische Universität Dresden, Dresden, Germany \\ $\mathrm{b}$ Insitute of Clinical Psychology and Psychotherapy, Department of Psychology, Technische Universität \\ Dresden, Dresden, Germany \\ c Neuroimaging Center, Department of Psychology, Technische Universität Dresden, Dresden, Germany
}

\begin{abstract}
Subjects participating in magnetic resonance imaging (MRI) examinations regularly report anxiety and stress related reactions. This may result in impaired data quality and premature termination of scans. Moreover, cognitive functions and neural substrates can be altered by stress. While prior studies investigated pre-post scan differences in stress reactions only, the present study provides an in-depth analysis of mood changes and hormonal fluctuations during the time course of a typical fMRI session. Thirty-nine subjects participated in the study. Subjective mood, salivary alpha-amylase (sAA) and cortisol were assessed at six time points during the lab visit. Associations between hormonal data and neural correlates of a visual detection task were observed using a region of interest approach applied to the thalamic region. Mood and hormonal levels changed significantly during the experiment. Subjects were most nervous immediately after entering the scanner. SAA was significantly elevated after MRI preparation. A subgroup of $n=5(12.8 \%)$ subjects showed pronounced cortisol responses exceeding $2.5 \mathrm{nmol} / 1$. Preliminary fMRI data revealed an association between sAA levels and left thalamic activity during the first half of the experiment that disappeared during the second half. No significant correlation between cortisol and thalamic activity was observed. Results indicate that an fMRI experiment may elicit subjective and neuroendocrine stress reactions that can influence functional activation patterns.
\end{abstract}

Keywords: fMRI, Alpha-amylase, Stress, Mood, Cortisol, Noradrenaline, Sympathetic nervous system, Thalamus

\section{Introduction}

Although affective response systems have been extensively studied within the last decade using functional magnetic resonance imaging (fMRI), surprisingly less is known about the stress-eliciting properties of the scanner itself. Anxiety-related reactions were often reported by routine care patients undergoing an MRI examination (Melendez and McCrank, 1993). The claustrophobic properties of the scanner, discomfort, loudness, a low sense of control and the novelty of the situation can act as stressful factors. Moreover, worries about innocuousness of the procedure or pathological incidental findings can result in anxiety and stress (Katz et al., 1994). These adverse reactions can be followed by impaired data quality and premature terminations of scans. 
Evidence is accumulating that anticipation of an MRI examination can affect subjective as well as neuroendocrine stress markers. Dantendorfer et al. (1997) found that state anxiety is significantly higher in pre- than post-scan measurements. Increased activation of the SAM as indicated by salivary alpha-amylase (sAA) was reported by van Stegeren et al. (2006) immediately before the scanning onset. In a repeated measurements design of six participants, Tessner et al. (2006) found that adult subjects with no previous scanning experience (e.g. scanner-naive subjects) showed an activation of the HPA axis as indicated by increased levels of salivary cortisol after scanning at the first day of examination, but not during repetition after one week. Similarly, in an examination of 160 adolescents Eatough et al. (2009) evaluated HPA and hypothalamic-pituitarygonadal (HPG) axis reactions via salivary cortisol, dehydroepiandrostosterone and testosterone. All three hormonal levels showed increases after an fMRI, but not after a simulation scan at a mock scanner. Moreover, the cognitive task being conducted in the scanner can also exhibit stress-eliciting properties. Kukolja et al. (2008b) reported that some of the subjects undergoing a cognitive challenging memory task in the scanner showed cortisol responses similar to those observed after psychosocial stress exposure such as the Trier Social Stress Test (TSST, Kirschbaum et al., 1993).

It is well known that stress acts upon various hormonal response systems, including the sympatho-adrenal-medullary (SAM) and hypothalamic-pituitary-adrenal (HPA) axis with glucocorticoids (CG) and catecholamines as their primary effectors. On a central nervous level, activation of the locus coeruleus noradrenaline (LC-NA) system and the solitary tract may influence tonic as well as phasic neuronal activity throughout the brain (Aston-Jones and Cohen, 2005; Valentino and van Bockstaele, 2008). Central NA binds to multiple sites such as amygdala, bed nucleus of the stria terminalis, prefrontal cortex, and lateral septum (Morilak et al., 2005). A high density of GC binding receptors is located in hippocampal and prefrontal structures (Reul and de Kloet, 1986). In accordance with these neuroanatomical findings, modulating effects of GC as well as NA were repeatedly demonstrated for a wide range of cognitive functions like working memory (Elzinga and Roelofs, 2005; Oei et al., 2006, 2009; Lupien et al., 2007; Schoofs et al., 2008; Weerda et al., 2010), declarative memory (de Quervain et al., 2000, 2003; Buchanan et al., 2006), emotional information processing and memory (van Stegeren et al., 2006; Wolf, 2008; Kukolja et al., 2008a), and temporal orienting (Coull et al., 2001). Several neuroimaging studies provide evidence for a relationship between NA effects and regions of the attention and arousal network, for example the thalamus (Kinomura et al., 1996; Coull et al., 2004; Bruhl et al., 2010). It is thus plausible to assume that stress induced by the MRI environment may influence functional activation patterns that are subject to neuroimaging research. Regarding clinical research it should moreover be anticipated that compared to healthy controls, specific patient groups vulnerable to the stress eliciting properties of the scanner (e.g. anxiety patients) may respond with enhanced stress reactions. Comparison of patient and control groups could hence be confounded by different stress levels.

In summary, first studies point towards the potentially stress-eliciting properties of the MRI environment. These unintended effects are especially important with regard to neuroimaging research where they could act as potential confounders of functional data. This demonstrates the need for a comprehensive examination of how different stress response systems may change during the time course of an fMRI session. Simple pre-post scanning differences as used in previous studies may be insufficient to detect different response latencies and hormonal changes throughout the entire fMRI session. The aim of the present study was to observe the extent to which subjective and bodily stress systems will get activated during an fMRI session and may in turn influence functional activation patterns of interest. In order to 
get more information about the scanning epoch itself, we implemented several assessment points during the experiment, including a mood questionnaire and hormonal measurements of sAA for SAM (Nater and Rohleder, 2009) and salivary cortisol for HPA reactivity

(Kirschbaum and Hellhammer, 1994). Based on previous findings about the potential stresseliciting effects of the MRI environment we predicted that subjective and hormonal stress components would be elevated in response to the scanner session, but not in response to a lab training session prior to the scanner session. Moreover, we predicted a relationship between hormonal levels and functional brain activation in structures subserving attention and arousal.

\section{Methods}

\subsection{Subjects and procedure}

Thirty-nine student volunteers from the Technische Universität Dresden (Germany) were recruited using flyers and public announcements (male: $\mathrm{n}=34(87.2 \%)$; mean age=23.8 $( \pm 2.1)$; female: $\mathrm{n}=5(12.8 \%)$; mean age $=23.8( \pm 4.8))$. Thirty-six subjects had never undergone an MRI or CT examination before yet (e.g. 92.3\% scanner-naive subjects). Exclusion criteria included smoking, oral contraceptives, body mass index above 26 , any history of acute or chronic medical disease or failure to meet basic MRI compatibility. Subjects received course credit or $10 €$ for participation. All participating subjects gave their informed consent, thus complying with the standards laid down in the Declaration of Helsinki. The study protocol was approved by the local ethics committee.

All assessments took place between 11:00 and 18:30, thus omitting morning times where cortisol levels show the most pronounced changes in diurnal rhythm. Subjects were asked to come well rested to testing and were also requested not to eat or drink one hour before the experiment. After entering the lab, subjects were informed about the study protocol and MRI procedure (see Fig. 1 for protocol details). To ensure that all participants had adequate levels of blood glucose they received $2 \mathrm{~d}$ grape juice immediately after the first saliva sample in order to avoid a hyporeaction of the HPA axis due to low glucose levels (Kirschbaum et al., 1997). We then conducted an extensive training session for the cognitive task consisting of 600 events in order to avoid further learning effects in the scanner. Afterwards subjects were introduced to the scanner room and prepared for the scanning session. The MRI protocol encompassed two experimental runs using the same paradigm that were separated by a structural scan. Following the scanning session subjects completed several questionnaires. During the entire lab visit we established six sampling points (T1-T6) for saliva and state affectivity, three of them being conducted while the subjects remained in the scanner (synthetic swabs being passed by an assistant). MRI staff was held constant for all subjects in order to avoid stress reactions due to differential personal styles and interactions.

\subsection{Measures}

\subsubsection{State and trait affectivity}

A mood profile from the entire lab visit was collected using the Multidimensional Mood Questionnaire (Mehrdimensionaler Befindlichkeitsfragebogen: MDBF, Steyer et al., 1994, 1997), which is suitable to measure short term fluctuations within several minutes. The MDBF in its long form consists of 24 items assessing three mood dimensions (valence: high valence: good mood, low valence: bad mood; alertness: high alertness: awake, low alertness: tired; calmness: high calmness: calm, low calmness: nervous). It can be divided into two 
parallel short forms that were alternated during the assessments. We programmed a computerized version of the MDBF using Presentation ${ }^{\circledR} 11.3$ (Neurobehavioral Systems, Inc., CA) that was used during the training session and at the scanner. Due to technical problems complete mood profiles could be saved for $n=36$ subjects.

After completion of the scanner session several questionnaires were used to characterize trait affectivity including depressive symptoms (Beck Depression Inventory: BDI II, Hautzinger et al., 2006) general anxiety (Anxiety Sensitivity Index: ASI, Hoyer and Markgraf, 2003; Stait Trait Anxiety Inventory-Trait Version: STAI, Laux et al., 1981), and claustrophobic anxiety (Claustrophobia Questionnaire: CLQ, Radomsky et al., 2001). In order to analyze differences in trait affectivity between hormonal subgroups, we subdivided the entire sample according to cortisol levels (cortisol responders and non-responders) and to SAA levels (median split procedure; see Section 2.2.4) and then compared markers of trait affectivity. BDI II and CLQ scores were available for $\mathrm{n}=38$ subjects.

\subsubsection{FMRI task, data acquisition and analysis}

A visual detection task similar to that used by Coull et al. (2000) was employed in order to study effects of the noradrenergic system (as indicated by sAA) on the attention network encompassing the thalamus. The task required to direct attentional resources to a particular time point. This simple detection task did not contain any stress eliciting properties; moreover, subjects received training prior to the fMRI session in order to assure constant performance levels. Baseline stimuli were two concentric circles with a small centered fixation cross presented foveally on a black background. Each trial started with a brightening $(100 \mathrm{~ms})$ of one of the circles indicating either a $600 \mathrm{~ms}$ (inner circle) or a $1400 \mathrm{~ms}$ (outer circle) cue-target interval (CTI) followed by the appearance $(100 \mathrm{~ms})$ of a large cross (target) superimposed over the baseline stimuli. Participants had to detect the target by pressing a button with the right index finger as fast as possible. Seven different trial types occurred in a pseudo-randomized order for blocks of 200 events. The total number of events per run was 400 with 235 valid trials (118 short cued, 117 long cued), 55 invalid trials ( 27 short cued, 28 long cued), 10 catch trials (5 short and long cues respectively) and 100 Null-Events (Josephs and Henson, 1999) were a baseline stimulus was shown. The randomized presentation of different trials and null-events leads to variable SOAs to prevent for stimulus-response predictability. During valid trials participants had to orient their attention to a time point 600 ms (short CTI) or $1400 \mathrm{~ms}$ (long CTI) after the presentation of the cue stimulus. During the invalid trials the CTI was reversed. This required a bottom-up driven re-orientation of attention when the target appeared to an unexpected early time point (exogen shifting) or a reorientation to an unexpected late time point under voluntary control (endogen shifting). During catch trials, only the cue but not the target was shown. Participants who reacted on the catch trials more than three times were excluded from the fMRI analysis. Each trial lasted $2000 \mathrm{~ms}$. The paradigm was programmed using Presentation ${ }^{\circledR} 11.3$ (Neurobehavioral Systems, Inc., CA). Stimuli were presented on video goggles (VisuaStim Digital, Northridge, California).

MRI images were acquired using a 3-Tesla Trio-Tim MRI wholebody scanner (Siemens, Erlangen, Germany). In each of the runs, 411 volumes of 38 slices with voxel size of $3 \times 3 \times 3$ $\mathrm{mm}$ (1 mm gap) were acquired sequentially. The first four scans were discarded to account for T1 equilibration effects. A standard 12 channel head coil and standard headphones were applied. Functional measurements were obtained using a T2* weighted gradient echo planar images (EPI) sequence (repetition time TR $2200 \mathrm{~ms}$, echo time TE $25 \mathrm{~ms}$ ). Structural images 
were obtained by using a Magnetization Prepared Rapid Gradient Echo Imaging (MPRAGE) sequence (repetition time TR $1900 \mathrm{~ms}$, echo time TE $2.26 \mathrm{~ms}$ ).

FMRI Data were analysed using SPM5 (Welcome Trust Centre for Neuroimaging, UCL, London, UK). Functional images of both runs were spatially realigned and unwarped to correct for movement artefacts followed by a correction for slice acquisition times. Structural images were coregistered to the functional scans and all volumes were normalized to the MNI (Montreal Neurological Institute, Quebec, Canada) reference brain. A smoothing kernel with $8 \mathrm{~mm}$ full-with half-maximum was used. At the first level regressors were built for the four effects of interest (valid short cue, valid long cue, invalid short cue, invalid long cue) and two effects of no interest (catch trial short, catch trial long). All regressors were modeled as single events and time locked to the onset of the cue. The general linear model was used to calculate regression coefficients (beta values) for the regressors and each voxel. The resulting contrast estimates were taken into the second level analysis employing a full factorial design. We expected sAA levels to rise directly prior to the first run. Since the NA system exhibits rather short response latencies with phasic, short lasting effects (Joels and Baram, 2009), we focused our analysis on the first run, subdividing it into two parts in order to investigate timedependent effects of the NA system. A region of interest (ROI) approach (ROI-size 1291 voxel) was applied to the thalamic region using the WFU Pickatlas (Maldjian et al., 2003). The estimated beta values were extracted from the peak voxel of the first half of run 1 employing the average effect of condition (significance threshold $p<0.01$ family wise error corrected for multiple comparisons). Associations between hormonal measures from saliva sample 3 and beta values from the four effects of interest were calculated using Pearson's correlations that were corrected for multiple comparisons (Bonferroni corrections; adjusted $\alpha=0.012$ ). Ten participants were excluded from the fMRI Data due to elevated movement parameters $(n=2)$, technical problems $(n=4)$, response to catch trials $(n=2)$ or unphysiological high sAA levels $(n=2)$.

\subsubsection{Hormonal determinations of cortisol and alpha-amylase}

Saliva samples were collected using Salivetten ${ }^{\circledR}$ (Sarstedt, Nümbrecht, Germany). Subjects were instructed to gently chew the swabs in order to stimulate saliva flow. Outside the scanner, swabs were directly passed to the subject by the study personnel. During the scanner session, subjects lying on the MRI table were put back into home position. Study personnel entered the scanner room and collected the sample, avoiding any movements for subjects. All saliva samples were stored $\mathrm{a}-20{ }^{\circ} \mathrm{C}$ until being assayed at the end of the study. Concentration of sAA was measured by an enzyme kinetic method: saliva was processed on a Genesis RSP8/150 liquid handling system (Tecan, Crailsheim, Germany). First, saliva was diluted 1:625 with double-distilled water by the liquid handling system. Twenty microliters of diluted saliva and standard were then transferred into standard transparent 96-well microplates (Roth, Karlsruhe, Germany). Standard was prepared from "Calibrator f.a.s." solution (Roche Diagnostics, Mannheim, Germany) with concentrations of 326, 163, 81.5, 40.75, 20.38, 10.19, and $5.01 \mathrm{U} / \mathrm{ml}$ alpha-amylase, respectively, and bidest water as zero standard. After that, 80 $\mathrm{ml}$ of substrate reagent (a-amylase EPS Sys, Roche Diagnostics, Mannheim, Germany) were pipetted into each well using a multichannel pipette. The microplate containing sample and substrate was then warmed to $371 \mathrm{C}$ by incubation in a waterbath for $90 \mathrm{~s}$. Immediately afterwards, a first interference measurement was obtained at a wavelength of $405 \mathrm{~nm}$ using a standard ELISA reader (Anthos Labtech HT2, Anthos, Krefeld, Germany). The plate was then incubated for another $5 \mathrm{~min}$ at $371 \mathrm{C}$ in the waterbath, before a second measurement at $405 \mathrm{~nm}$ was taken. Increases in absorbance were calculated for unknowns and standards. Increases of absorbance of diluted samples were transformed to alpha-amylase concentrations using a 
linear regression calculated for each microplate (Graphpad Prism 4.0c for MacOSX, Graphpad Software, San Diego, CA). Inter- and intra-assay variation was below 10\%. Analysis of cortisol concentrations was carried out by means of salivary cortisol immunoassay with time-resolved fluorescence endpoint detection. All samples were run in duplicate in the run on the same kit. Cortisol levels were expressed in nmol/l, internal and external controls were included in the assays. The intra- and inter-assay variation coefficients ranged between $4.0-6.7 \%$ and $7.1-9.0 \%$, respectively. All analyses were conducted at the psychoendocrinological laboratory, Chair of Biopsychology, Technische Universität Dresden.

\subsubsection{Data parameterization}

In addition to profile plots of state affectivity and hormonal changes from the entire lab visit we calculated peak measures for mood dimensions, sAA and cortisol during the scanner session (T3-T6). In order to compare high and low hormonal responders regarding trait characteristics, baseline to peak values (BL-Peak) were computed. Assuming a certain habituation time to the lab environment we employed T2 as baseline value and subtracted it from the MRI peak measure. Subjects exceeding cortisol BL-Peak values of $2.5 \mathrm{nmol} / 1$ were classified as responders (Wust et al., 2000). Since comparable conventions are not available for sAA, we used a median split procedure on the BL-Peak value in order to distinguish between high and low sAA level groups. SAA samples from T1 to T4 were missing for one subject, so that BL-Peak values were calculated for $n=38$ subjects. In case of single missing values (insufficient saliva samples cortisol: $n=2$; abnormally high sAA: $n=3$ ) we used a linear interpolation from the pre- and proceeding values to calculate an estimate.

\subsubsection{Statistical analysis}

Mood and hormonal changes were tested using a one-factorial analysis of variance (ANOVA) with repeated measurements on the factor "sampling point" and subsequent pairwise comparisons. Greenhouse-Geisser adjustments were applied when appropriate. Partial eta2 were transformed into Cohen's $\mathrm{f}^{2}$ as effect size measure $(0.10=$ small effects; $0.25=$ medium effects; 0.40=large effects; Cohen, 1983). Associations between state affectivity and hormonal measures were calculated using Pearson's correlations that were corrected for multiple comparisons (Bonferroni corrections; adjusted $\alpha=0.007$ ). Differences between cortisol responders vs. non-responders and sAA high vs. low level groups were analyzed using a two-factorial ANOVA with repeated measurements on the factor "sampling point" and the between-subjects factor "group". We used this ANOVA approach in order to test for systematic differences between subgroups that were not specific for the scanner session (e.g. baseline differences or different hormonal levels outside the scanner session time-window). Demographic characteristics and state and trait affectivity were tested using chi ${ }^{2}$, student's ttests for independent samples. Non-parametric tests (Mann-Whitney U-test) where used when appropriate.

\section{Results}

\subsection{Mood dimensions}

As displayed in Fig. 2, all three mood dimensions changed significantly during the lab visit (valence: $F(5,175)=6.15 ; \mathrm{p}<0.001 ; \mathrm{f}^{2}=0.42$; calmness: $\mathrm{F}(5,175)=5.31 ; \mathrm{p}<0.001 ; \mathrm{f}^{2}=0.39$; alertness: $\left.F(3,122)=24.08 ; \mathrm{p}<0.001 ; \mathrm{f}^{2}=0.83\right)$. Pairwise comparisons showed that subjects reported lower valence ratings after the training session. This level remained unchanged 
during MRI preparation and the first run. After the structural scan, however, valence significantly improved, but went down again after the second run (valence: T1 $>\mathrm{T} 2, \mathrm{~T} 3, \mathrm{~T} 4$ $\mathrm{p}<0.050 ; \mathrm{T} 5>\mathrm{T} 2, \mathrm{p}<0.010 ; \mathrm{T} 5>\mathrm{T} 3, \mathrm{~T} 4, \mathrm{~T} 6, \mathrm{p}<0.001)$. A similar course was observed for calmness ratings with subjects being most nervous immediately before the first run (calmness: $\mathrm{T} 1>\mathrm{T} 3, \mathrm{p}<0.050 ; \mathrm{T} 5>\mathrm{T} 2, \mathrm{p}<0.050 ; \mathrm{T} 5>\mathrm{T} 3, \mathrm{~T} 4, \mathrm{p}<0.001 ; \mathrm{T} 6>\mathrm{T} 3, \mathrm{~T} 4, \mathrm{p}<0.010$ ). In contrast, alertness ratings exhibited a differential course. Subjects reported feeling more tired after the cognitive task (either training session or MRI run; alertness: T1, T3, T5 $>\mathrm{T} 2$, T4, T6, $\mathrm{p}<0.001$; $\mathrm{T} 3>\mathrm{T} 5, \mathrm{p}<0.050)$.

Peak valence values, but not calmness or alertness were negatively correlated with peak cortisol levels during the MRI session (valence/ cortisol: $r=-.45, \mathrm{p}=0.004$; calmness/cortisol: $\mathrm{r}=-0.233$, ns; alertness/ cortisol: $\mathrm{r}=-0.135$, ns). Peak sAA levels, on the other hand, were not significantly associated with any of the mood dimensions or with cortisol levels (valence/sAA: $r=0.092$, ns; calmness/sAA: $r=0.168$, ns; alertness/sAA: $r=0.174$, ns; cortisol/sAA: $\mathrm{r}=0.201$, ns).

\subsection{Neuroendocrine data}

Changes in hormonal levels during the lab visit are displayed in Fig. 3. ANOVA results substantiated significant changes of sAA and cortisol values (alpha-amylase: $F(3,124)=5.76$; $\mathrm{p}=0.001 ; \mathrm{f}^{2}=0.40$; cortisol: $\left.\mathrm{F}(2,66)=6.86 ; \mathrm{p}=0.003 ; \mathrm{f}^{2}=0.43\right)$. Pairwise comparisons showed that sAA peaked directly before the first run after subjects had entered the scanner (T3) and then again after the second run (T6; T3 $>\mathrm{T} 5, \mathrm{p}<0.001$; T3 $>\mathrm{T} 1, \mathrm{~T} 2, \mathrm{~T} 4, \mathrm{~T} 6, \mathrm{p}<0.01$; T5bT4, T6, $\mathrm{p}<0.001)$. Cortisol levels, however, showed no significant MRI related rise, but a general falling trend $(\mathrm{T} 1>\mathrm{T} 3, \mathrm{p}<0.001 ; \mathrm{T} 1>\mathrm{T} 2, \mathrm{~T} 5, \mathrm{~T} 6, \mathrm{p}<0.01 ; \mathrm{T} 1>\mathrm{T} 4, \mathrm{p}<0.050 ; \mathrm{T} 2>\mathrm{T} 3, \mathrm{p}<0.001$; $\mathrm{T} 2>\mathrm{T} 6, \mathrm{p}<0.050 ; \mathrm{T} 6 \mathrm{bT} 4, \mathrm{~T} 5, \mathrm{p}<0.010)$.

We furthermore analyzed differences between sAA high vs. low groups. As expected, ANOVA results on sAA levels indicated a main effect of sampling point and a main group effect (sampling point: $F(4,136)=7.86, p<0.001, f^{2}=0.46$; group: $F(1,35)=16.02, p<0.001$, $\left.\mathrm{f}^{2}=0.68\right)$. While the main effect of group can be explained by the median split procedure, we furthermore observed an interaction effect of group and sampling point, indicating different sAA levels at the respective time points in each group. This interaction effect showed comparable values during baseline at $\mathrm{T} 2$, but significantly increased values throughout the entire scanning session (group*sampling point: $(\mathrm{F} 4,136)=9.23 ; \mathrm{pN} 0.001 ; \mathrm{f}^{2}=0.51 ; \mathrm{T} 3, \mathrm{~T} 4, \mathrm{~T} 5$, T6: low level group-high level group, $\mathrm{p}<0.001$; T1: low level group $<$ high level group, $\mathrm{p}<0.01)$. Moreover, within-subject comparisons showed that the low level subjects were lacking a scanner-related peak in sAA as evidenced by a general falling trend over time (low level group: $\mathrm{T} 1>\mathrm{T} 5, \mathrm{p}<0.05$; T2 $>\mathrm{T} 4, \mathrm{~T} 6, \mathrm{p}<0.05$; $\mathrm{T} 2>\mathrm{T} 5, \mathrm{p}<0.01$; $\mathrm{T} 3>\mathrm{T} 6, \mathrm{p}<0.05$ ). In contrast, only high sAA level subjects showed a pronounced increase in sAA to T3 and again to T6 (high level group: T3>T1, T2, T5, pN0.001; T3 $>$ T4, T6, p<0.01; T4 $>\mathrm{T} 2, \mathrm{p}<0.001$; $\mathrm{T} 4>\mathrm{T} 5, \mathrm{p}<0.01 ; \mathrm{T} 4>\mathrm{T} 1, \mathrm{p}<0.05 ; \mathrm{T} 5>\mathrm{T} 2, \mathrm{p}<0.05 ; \mathrm{T} 6>\mathrm{T} 2, \mathrm{p}<0.001 ; \mathrm{T} 6>\mathrm{T} 5, \mathrm{p}<0.05)$.

Differences between cortisol responders and non-responders yielded similar results. While the main effect of group remained insignificant, ANOVA results showed a main effect of sampling point and an interaction effect of sampling point * group (group: $F(1,37)=2.29$, $\mathrm{p}=0.138 ; \mathrm{f}^{2}=0.25$ ); sampling point: $\mathrm{F}(1,75)=4.64, \mathrm{p}=0.12, \mathrm{f}^{2}=0.35$; sampling point* group: $\left.\mathrm{F}(2,75)=18.52, \mathrm{p} b 0.001, \mathrm{f}^{2}=0.71\right)$. Responders exhibited elevated cortisol levels throughout the scanner session (T4, T5, T6: non-responders $<$ responders, $\mathrm{p}<0.001)$ but were comparable with non-responders for initial and baseline values. Within-subject comparisons affirmed a lacking peak in non-responders $(\mathrm{T} 1>\mathrm{T} 2, \mathrm{p}<0.001$; $\mathrm{T} 1, \mathrm{~T} 2>\mathrm{T} 3, \mathrm{~T} 4, \mathrm{~T} 5, \mathrm{~T} 6, \mathrm{p}<0.001$; $\mathrm{T} 3$, 
$\mathrm{T} 4>\mathrm{T} 5, \mathrm{~T} 6, \mathrm{p}<0.01 ; \mathrm{T} 5>\mathrm{T} 6, \mathrm{p}<0.01)$. Contrary, responders exhibited a pronounced peak from T4 to T6 compared to pre-scanner values (T1, T2bT4, T5, p<0.01; T1, T2bT6, p<0.05; $\mathrm{T} 3>\mathrm{T} 4, \mathrm{~T} 5, \mathrm{p}<0.001 ; \mathrm{T} 3>\mathrm{T} 6, \mathrm{p}<0.01)$. Three out of five cortisol responders were also in the SAA high level group. Due to the limited number of cortisol responders $(n=5)$ we also applied non-parametric tests for between and within-subject comparisons. These yielded comparable results.

\subsection{Correlation between hormonal levels and functional brain activation in the thalamus}

The ROI analysis of the thalamic region revealed a time sensitive association for the first, but not the second half of the first run between sAA and the BOLD signal for the "invalid short cue" trials (endogen shifting condition) within the left thalamus ( $x=-15, y=-18, z=9 ; Z=6,84$; 81 voxels; see Fig. 4). We found a significant correlation for beta values and sAA during the first seven minutes (e.g., 200 scans), that disappeared during the second half of run 1 . No significant correlation was observed with regard to the entire run (sAA/ 1 st half: $r=0.477$, $\mathrm{p}=0.009$; $\mathrm{sAA} / 2$ nd half: $\mathrm{r}=0.021$, ns; sAA/entire run: $\mathrm{r}=0.212$, ns). Associations between beta values from endogen shifts and cortisol revealed no significant results (cort/ 1 st half: $r=0.111$, ns; cort/2nd half: $r=-0.362$, ns; cort/entire run: $r=-0.183$; ns).

\subsection{Demographic variables and trait/state affect}

In the next step we aimed to test for psychological markers that could differentiate high from low hormonal subgroups. As can be seen in Tables $1 \mathrm{a}$ and $1 \mathrm{~b}$, neither cortisol nor sAA subgroups differed from each other in demographic variables or mood during the scanning session (indicated by mood dimension peak values). Cortisol responders showed significantly lower BDI II scores, although both groups were markedly below clinical cut-offs (e.g. BDI IIN18). Comparable data were also obtained for trait anxiety and claustrophobic fear for hormonal subgroups.

\section{Discussion}

Present results show that mood changed significantly during the time course of the assessment with subjects being most nervous immediately after entering the MRI. While valence and calmness ratings showed similar changes, alertness covaried with the amount of cognitive demand. Regarding hormonal reactions, the entire group showed a significant rise in sAA in response to the scanner. In contrast, cortisol values did not increase in general. However, five $(12.8 \%)$ subjects reacted with a pronounced cortisol response that continued even beyond the end of the scanner session. Peak cortisol values were negatively correlated with peak valence during the scanner session. We furthermore observed an association between sAA levels and functional brain activation being particularly pronounced during the first, but not the second half of run 1 . Hormonal responders and non-responders were largely comparable in affective trait markers.

\subsection{Mood and hormonal changes}

Regarding mood fluctuations, we observed differential changes in the dimensions studied, pointing towards the incremental value of multidimensional mood assessments compared to anxiety ratings only. Subjects were most nervous when entering the scanner. This appears to reflect the well known impression verbalized by subjects that participate in an MRI examination for the first time. Strong variations were also found in the alertness dimension 
with subjects getting fatigued after performing the visual detection task, regardless of being in the scanner or not. Alertness thus varied with performing the task and is likely to reflect effects of the cognitive load. From all dimensions, only valence correlated with peak cortisol levels during the session.

We cannot exclude the possibility that mood fluctuations are nonspecific for the MRI situation, but rather occur in experimental sessions per se. Mood changes in the scanner as evidenced by present results could nevertheless influence brain functions. Mood induction is a frequent technique used in neuroimaging studies to provoke affective responses (Schneider et al., 1994). Unintended mood inductions by the scanner setting itself should thus be taken into consideration when interpreting functional activation patterns. On the other hand, mood appeared to recover during the structural scan. When designing an fMRI experiment, the recreational potential of breaks from the cognitive task should be taken into consideration.

Although no stressor task was involved, sAA levels as an indicator of sympathetic activation increased in the entire group. SAA levels were highest during the MRI preparation phase. It could be argued, however, that increases in sAA have been influenced by movements (walking from the assessment to the scanner room), but this assumption is not supported by subgroup analyses. Although all subjects had comparable levels of physical activity only those in the high median group showed an increase in sAA. Thus, we conclude that rises in sAA levels are specific for the anticipation of entering the scanner. The high median group furthermore showed a second peak in SAA at T6. Since subjects maintained in the scanner from T3 to T6, the second peak is not likely to be influenced by physical activity, but rather appears to solely reflect psychological processes. Anticipating the next run, subjects may have aroused themselves after the structural scan.

Regarding HPA axis activation, our results do not demonstrate cortisol responses towards the scanner per se. Different results reported by other authors may be ascribed to differences in sample size ( $\mathrm{n}=6$, Tessner et al., 2006) or characteristics (e.g. adolescents, Eatough et al., 2009), differences in sampling points (e.g. pre-post measures only), duration of the scanner session or technical equipment. In this study, goggles were used for visual presentation. This may have reduced the claustrophobic properties of the tomograph. The type of stimulation system was not reported in previous studies, comparison remains thus limited. However, a subset of subjects (12.8\%) from the present sample showed pronounced reactions that were comparable to social stressors such as the TSST. In contrast to the SNS that is activated by a broad range of unspecific stimuli, the HPA axis responds to specific stressors such as social evaluation, novelty of the situation and low sense of control (Dickerson and Kemeny, 2004). We were not able to find differences in affective trait markers (general anxiety, claustrophobic anxiety) between high and low responders. However, the scanner environment may have been interpreted in many different ways by the subjects, especially with regard to HPA axis relevant features. Due to the passiveness during the scan and high degree of technization some, but not all subjects may have experienced a low sense of control in the scanner. Future studies should investigate subjective scanner experiences in more detail.

Moreover, present results show that hormonal changes may follow a different time course during the scanning session. For example, sAA levels peaked immediately before the first functional run, while cortisol levels in responders peaked during the second run. Thus, functional activation patterns from the first and second run may have been differently influenced by these two hormonal systems. Studies depending on pre-post differences only may fail to detect these changes that are related to different response latencies of the 
respective system. Before averaging several runs, the impact of changing hormonal levels during the scanner session should be evaluated.

We only observed an incomplete overlap between cortisol and sAA subgroups since two out of five cortisol responders were in the low median sAA group. A rise in cortisol may thus not necessarily be accompanied by activation of the amylase system. On the other hand, interactions of both systems on emotional processing and memory performance were reported by Kukolja et al. (2008a) and van Stegeren et al. (2010). However, the limited number of cortisol responders does not allow for a comprehensive analysis of covariances between these two systems.

\subsection{Interaction between stress hormones and functional activation patterns}

As already pointed out, several cortical and subcortical structures that are involved in cognitive and affective processes, including prefrontal structures, hippocampi and amygdalae, exert a high density of GC-binding receptors (Reul and de Kloet, 1986). Importantly, behavioral effects may depend on the binding ratio of gluco- (GR) and mineralcorticoid (MR) receptors. Basal HPA activity is associated with MR receptor binding, while phasic cortisol secretion additionally affects GR receptors (de Kloet et al., 1998). Depending on the amount of cortisol reactivity, stress responses related to the MRI setting may in some, but not all, subjects modulate neural activity. As a consequence, interindividual variance in imaging data due to different hormonal changes should be anticipated. SAA as an indicator of noradrenergic and sympathetic activity is also known to affect cognitive processes and neural correlates (McGaugh and Roozendaal, 2002; van Stegeren et al., 2005). The potential impact of elevated sAA levels during an fMRI experiment has recently been pointed out by van Stegeren et al. (2010). They reported a missing effect of enhanced memory after administrating the NA agonist yohimbine compared to a placebo condition. Authors discussed that elevated sAA levels in response to the scanning procedure within the drug as well as in the placebo group could be accountable for the missing effect. We presented preliminary fMRI data that further underline this hypothesis. To test for short lasting NA effects, the first run was split in two halves. During the first half, sAA was significantly correlated with left medial thalamic activity during the invalid trials where the target appeared to an unexpected late time point (endogen shifting); the other conditions were not influenced by NA activity. This is in line with Coull et al. (2001) where the NA $\alpha 2$ agonist Clonidine impaired only endogenous re-orientation, but not exogenous shifts or temporal orienting per se. Associations between the left medial thalamic activity and high NA levels were also reported by Bruhl et al. (2010). We did however not observe a correlation for the second half of the first run. This parallels findings from behavioral and neuroendocrine data where both sAA levels as well as subjective experience of stress peaked prior to the start of the session, but were already decreased by the end of first run. These results point towards time-sensitive influences of the adrenergic system on attentional functions. Present findings did not show any associations between thalamic activity and the cortisol system. Several reasons may account for the absence of correlations. First, the HPA axis exhibits longer response latencies than the short reacting adrenergic system. Indeed, peak values were observed after the first run. It may thus be plausible that cortisol values were rising during the first run, while sAA levels already peaked in the beginning. Second, pronounced cortisol reactions were observed in a relatively small number of subjects only, showing no effects for the entire group. Finally, the temporal orienting task was specifically designed to detect effects of the noradrenergic system. Hence, the task may have been not sensitive to cortisol effects, since these have been demonstrated for working memory and memory functions (Lupien et al., 2007; Kukolja et al., 2008b; Weerda et al., 2010). It may be a valuable task for future studies to employ tasks that are more 
sensitive to cortisol effects and to study the association between these hormonal parameters and functional activation patterns of interest in more detail.

\subsection{A priori identification of hormonal responders}

In order to identify hormonal responders from non-responders, we compared affective trait markers such as depressive symptoms, anxiety sensitivity or claustrophobic anxiety. Results however, were comparable to a large extent in both groups. Cortisol non-responders showed higher BDI values. Since scores were markedly below clinical cut-offs, these cannot be interpreted in terms of clinically relevant depressive symptoms. Other authors reported a predictive value of claustrophobic anxiety for subjective distress during the MRI or a mock assessment (McIsaac et al., 1998; McGlynn et al., 2003, 2007). We did not find such an association in the present sample. Future studies are needed to evaluate the predictive value of affective trait markers for the experience of MRI related distress.

\subsection{Limitations}

Results, however, have to be interpreted within the methodological limits of the study.

We cannot exclude the possibility that stress reactions are nonspecific for the MRI situation, but rather occur in experimental sessions per se. Although no group comparisons employing a second control condition (such as a lab session compared to a scanner session) are available, indirect inference for the specificity of MRI-related stressor effects can be derived from within-subject control conditions. Although all subjects underwent a lab training session prior to the scanner session, significant hormonal changes where observed during the scanner session only. Future studies are nevertheless needed that directly compare the impact of experiments in- and outside the scanner. Regarding the small amount of cortisol responders, a larger sample size would have been preferable, especially for closer characterization of this subgroup. Although we carefully screened for previous scanner experience, in a post scanning interview three subjects reported having been in a scanner before. The small size of scannerexperienced subgroup does not allow for further statistical analyses on this potential factor of influence. Although scanner-naive and -experienced subjects were equally distributed in the hormonal subgroups, future studies are clearly needed to investigate the effect of previous scanner experience on stress reactions towards the MRI environment. The selection of questionnaires used in this study did not contribute to an a priori characterization of hormonal responders; future studies should evaluate the potential benefit of additional markers such as controllability of the situation. In order to obtain a high level of internal validity, rather strict exclusion criteria were employed, including oral contraceptives, body mass index or any history of acute or chronic medical disease. As a consequence, our sample predominantly consisted of young and healthy male subjects, reflecting the typical study participant of fMRI experiments. The small number of cortisol responders may have been due to these sample characteristics; present results may be representative for this kind of studies only.

\subsection{Conclusion}

In conclusion, we were able to show that the MRI setting can act as a stressor. Subjects undergoing a cognitive task in the MRI environment showed a rise in sAA-levels directly before scanning onset, but no cortisol responses in general. However, these effects may vary due to the population studied (e.g. gender, age, familiarity to the scanner or psychiatric characteristics). As already stated in the Introduction, investigation of specific patient groups may result in more pronounced stress reactions. Clinical studies should therefore take into 
account that comparison of patient and control groups could be confounded by different stress levels.

We showed that stress reactions could serve as an additional source of interindividual variance in behavioral and neural data. Future studies are needed to evaluate the impact of these confounding variables in functional imaging studies in more detail. How can we deal with these unintended reactions? We tried to identify vulnerable subjects; however, first attempts were not successful yet. Since it may be difficult to exclude stress responders a priori, it is recommended to record intra- and interindividual stress levels in order to integrate data as covariates in the fMRI design model. An alternative approach might be to implement training activities that familiarize subjects with the scanner and thus reduce stress reactivity. This may help obtaining more homogenous hormonal levels in fMRI samples.

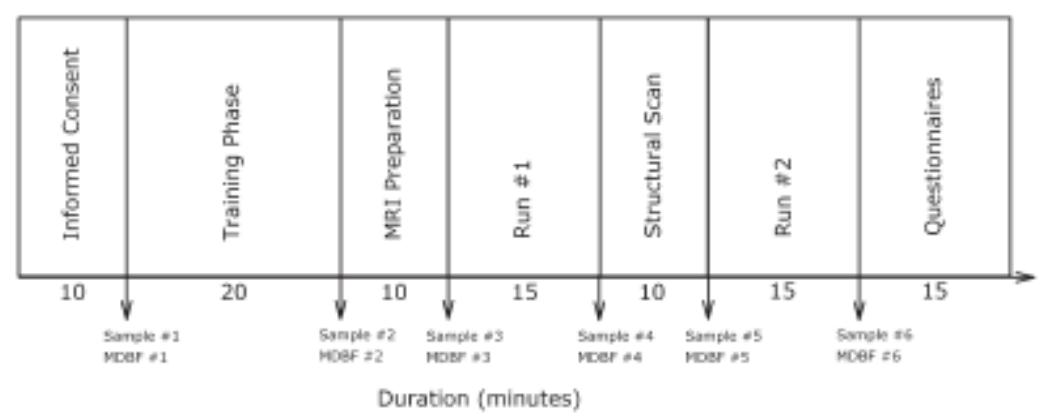

Fig 1. Study probcol. Rur: functional scan; Sumple: salivary smple, MDHF: Multidimentsional Mood Questionnaire.

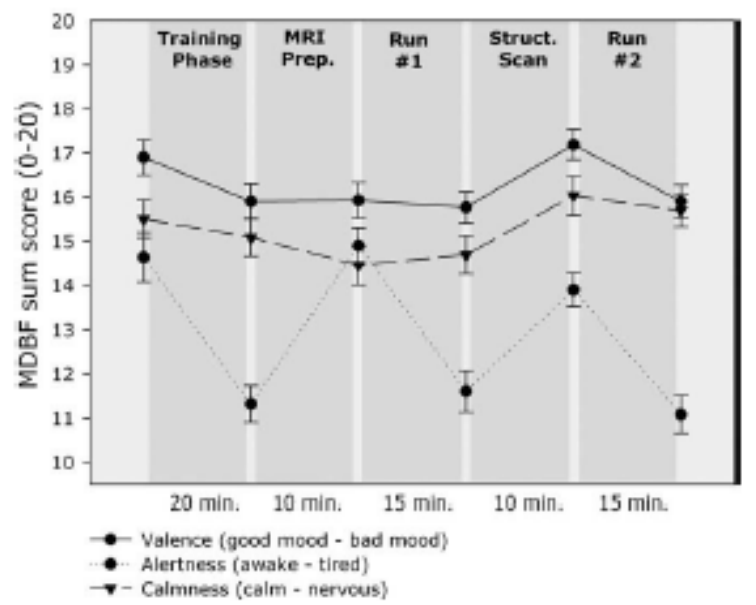

Fig. 2 Mood profiles during the lab visit Error hars indicats SEM Mood dimensians were razed on a five paint sale ranging from $1=$ not $x$ all to $5=$ very much. Valence: high valesice: good mood, low valence: had mood; alentness: high alertiess: awale, law alentness: tired; calmnes: high calmness: cal m, low calmness: nervour. MDHF: Multidimentional Mood Questionnuire; run: functionnal scan. 

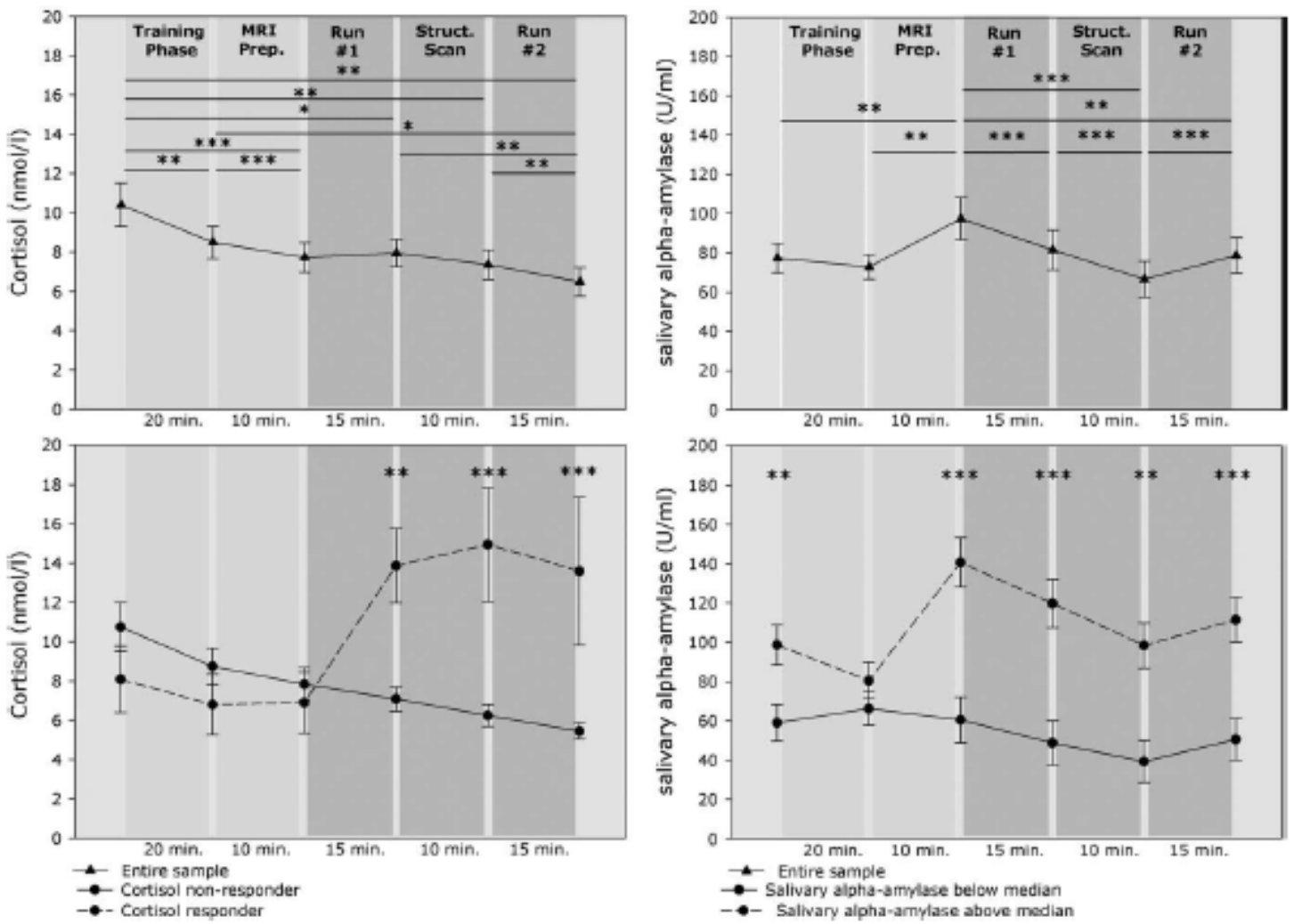

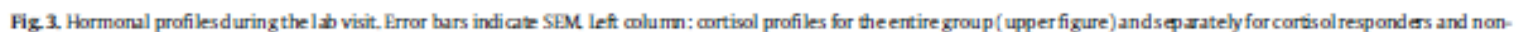
responders (lower figure). Right column: salivary alpha-anylase profiles for the entire group (upper figure) and for subjects above va below the median (lower figure). For the entire group, pairwise comparisons indicats changes over time; while for hormonal subgroups, pairwise comparisons indicate dhanges between groups (for alditional withinsubject changes pleze see results section). * $p<0.05 ; " * p<0.01 ; \cdots p<0.001$.
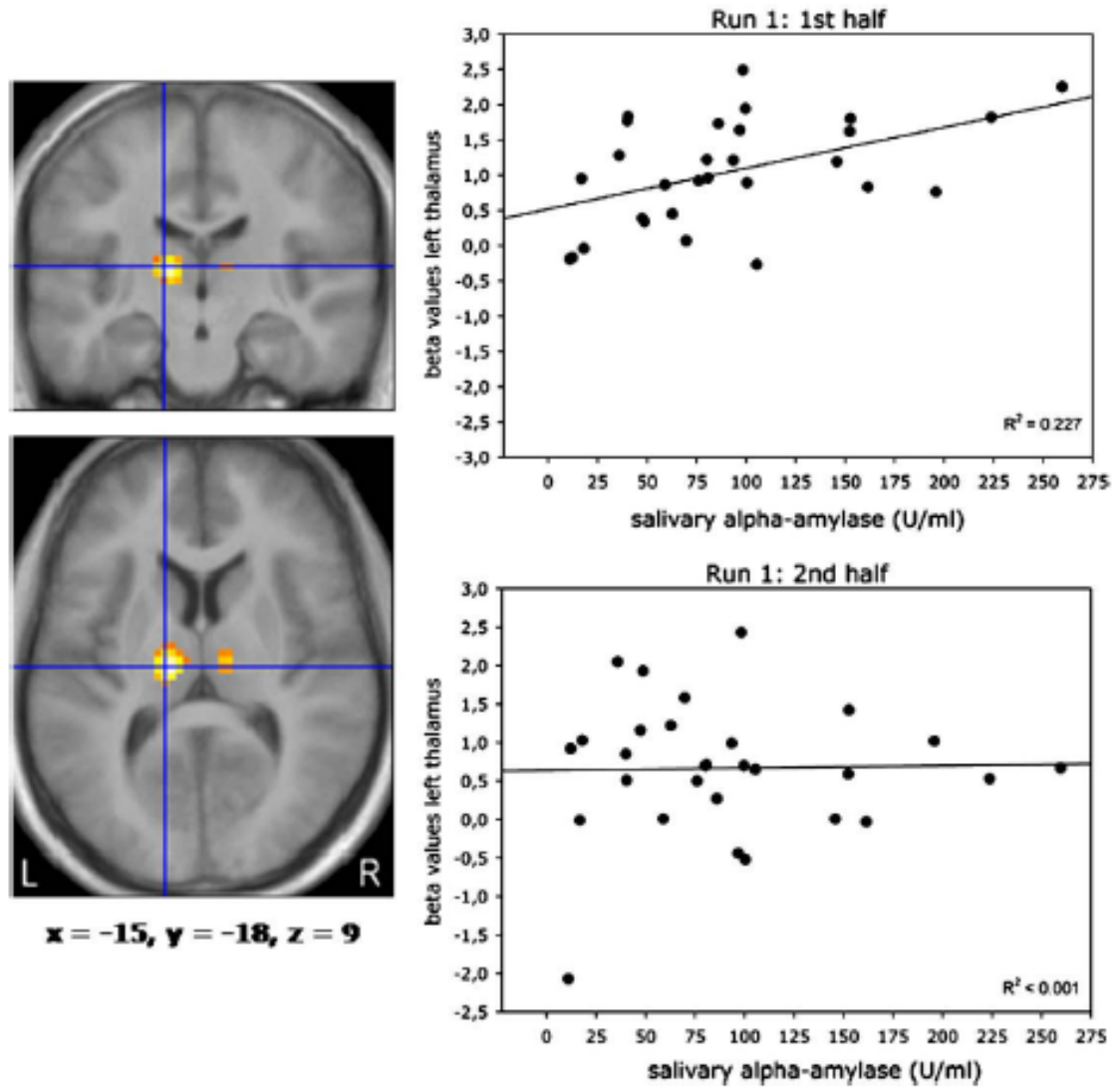

Fig.4. ROI analysis of the thalamic region for the first half of the first run. BOl-size 1291 Vaxek. Resuls neported are significant at $p<0.1$ (fimily wiseerror corrested for multiple comparisars ) - Graphs: Corrd tions between the beta val ues from the peak vowel and sAA smple 3. Upper gaph: 1st half of run 1. Lower graph: 2 nd half of run 1. 


\begin{tabular}{|c|c|c|c|c|c|}
\hline \multirow{3}{*}{$\begin{array}{l}\text { Male gender }[n(x)] \\
\text { Sanner-naive }[n(x)]\end{array}$} & \multicolumn{2}{|c|}{$\begin{array}{l}\text { Below median } \\
(n=20)\end{array}$} & \multicolumn{2}{|c|}{$\begin{array}{l}\text { Above median } \\
(n=18)\end{array}$} & \multirow{3}{*}{$\begin{array}{l}\vec{p}^{n} \\
\mathrm{~ns} \\
\mathrm{~ns}\end{array}$} \\
\hline & 17 & $(85.0)$ & 16 & (889) & \\
\hline & 18 & $(900)$ & 17 & (944) & \\
\hline Age [years] & 23.7 & (24) & 24.1 & $(27)$ & ns \\
\hline Time [hh $\mathrm{mm}]^{\mathrm{b}}$ & 1356 & $(\omega 2: 26)$ & $13: 46$ & (01:35) & ns \\
\hline \multicolumn{6}{|l|}{ State of ort } \\
\hline Peak valence & 17.4 & (1.9) & 173 & (1.9) & ns \\
\hline Peak calmness & 16.6 & (19) & 173 & (22) & ns \\
\hline Peak alertiess & 153 & (23) & 15.5 & (22) & ns \\
\hline \multicolumn{6}{|l|}{ Trait affect } \\
\hline BDI II & 38 & $(7.0)$ & 44 & $(3.7)$ & ns \\
\hline ASI & 18 & (1.7) & 1.6 & (1.9) & ns \\
\hline STAI & 47.6 & (1.8) & 473 & (1.8) & ns \\
\hline $\mathrm{CQQ}$ & 149 & (125) & 10.9 & (81) & ns \\
\hline
\end{tabular}

\begin{tabular}{|c|c|c|c|c|c|}
\hline \multirow[b]{2}{*}{ Male gender $[n(X)]$} & \multicolumn{2}{|c|}{$\begin{array}{l}\text { Nan-responders } \\
(n=34)\end{array}$} & \multicolumn{2}{|c|}{$\begin{array}{l}\text { Responders } \\
(n=5)\end{array}$} & \multirow{2}{*}{$\frac{p^{n}}{\mathrm{~ns}}$} \\
\hline & 29 & $(85.2)$ & 5 & $(1000)$ & \\
\hline Scanner-nuive [n (x)] & 31 & (91.2) & 5 & $(1000)$ & ns \\
\hline Age [years] & 239 & (26) & 23.4 & (21) & ns \\
\hline Time [hh $m m]^{b}$ & 140102 & $(\propto 2: 15)$ & $13: 36$ & $(02: 32)$ & ns \\
\hline \multicolumn{6}{|l|}{ Stute offert ${ }^{<}$} \\
\hline Peak valence & 173 & (1.7) & 16.4 & (24) & ns \\
\hline Peak calmness & 169 & (20) & 16.4 & (25) & ns \\
\hline Peak alertiess & 154 & (23) & 15.0 & (21) & ns \\
\hline \multicolumn{6}{|l|}{ Trait offert } \\
\hline BDIII & 49 & (60) & 06 & (as) & 0.004 \\
\hline ASI & 18 & (19) & 1.0 & (1.2) & ns \\
\hline STAI & 47.4 & (18) & 47.6 & (1.1) & ns \\
\hline $\mathrm{ClQ}$ & 134 & (10.8) & 10.4 & (10.1) & ns \\
\hline \multicolumn{6}{|c|}{ 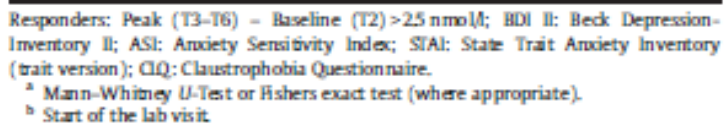 } \\
\hline
\end{tabular}

\section{References}

- Aston-Jones, G., Cohen, J.D., 2005. An integrative theory of locus coeruleusnorepinephrine function: adaptive gain and optimal performance. Annu. Rev. Neurosci. 28, 403-450.

- Bruhl, A.B., Kaffenberger, T., Herwig, U., 2010. Serotonergic and noradrenergic modulation of emotion processing by single dose antidepressants. Neuropsychopharmacology 35, 521-533.

- Buchanan, T.W., Tranel, D., Adolphs, R., 2006. Impaired memory retrieval correlates with individual differences in cortisol response but not autonomic response. Learn. Mem. 13, 382-387.

- Cohen, J., 1983. Statistical power analysis for the behavioral sciences. Lawrence Erlbaum, Hillsdale.

- Coull, J.T., Frith, C.D., Buchel, C., Nobre, A.C., 2000. Orienting attention in time: behavioural and neuroanatomical distinction between exogenous and endogenous shifts. Neuropsychologia 38 (6), 808-819.

- Coull, J.T., Nobre, A.C., Frith, C.D., 2001. The noradrenergic alpha2 agonist clonidine modulates behavioural and neuroanatomical correlates of human attentional orienting and alerting. Cereb. Cortex 11, 73-84.

- Coull, J.T., Jones, M.E., Egan, T.D., Frith, C.D., Maze, M., 2004. Attentional effects of noradrenaline vary with arousal level: selective attention of thalamic pulvinar in humans. Neuroimage 22, 315-322.

- Dantendorfer, K., Amering, M., Bankier, A., Helbich, T., Prayer, D., Youssefzadeh, S., Alexandrowicz, R., Imhof, H., Katschnig, H., 1997. A study of the effects of patient anxiety, perceptions and equipment on motion artifacts in magnetic resonance imaging. Magn. Reson. Imaging 15 (3), 301-306.

- de Kloet, E.R., Vreugdenhil, E., Oitzl, M.S., Joels, M., 1998. Brain corticosteroid receptor balance in health and disease. Endocr. Rev. 19 (3), 269-301.

- de Quervain, D.J., Roozendaal, B., Nitsch, R.M., McGaugh, J.L., Hock, C., 2000. Acute cortisone administration impairs retrieval of long-term declarative memory in humans. Nat. Neurosci. 3 (4), 313-314. 
- de Quervain, D.J., Henke, K., Aerni, A., Treyer, V., McGaugh, J.L., Berthold, T., Nitsch, R.M.,

- Buck, A., Roozendaal, B., Hock, C., 2003. Glucocorticoid-induced impairment of declarative memory retrieval is associated with reduced blood flow in the medial temporal lobe. Eur. J. Neurosci. 17 (6), 1296-1302.

- Dickerson, S.S., Kemeny, M.E., 2004. Acute stressors and cortisol responses: a theoretical integration and synthesis of laboratory research. Psychol. Bull. 130 (3), 355-391.

- Dressendorfer, R.A., Kirschbaum, C., Rohde, W., Stahl, F., Strasburger, C.J., 1992. Synthesis of a cortisol-biotin conjugate and evaluation as a tracer in an immunoassay for salivary cortisol measurement. J. Steroid Biochem. Mol. Biol. 43 (7), 683-692.

- Eatough, E.M., Shirtcliff, E.A., Hanson, J.L., Pollak, S.D., 2009. Hormonal reactivity to MRI scanning in adolescents. Psychoneuroendocrinology 34 (8), 1242-1246.

- Elzinga, B.M., Roelofs, K., 2005. Cortisol-induced impairments of working memory require acute sympathetic activation. Behav. Neurosci. 119 (1), 98-103.

- Hautzinger, M., Keller, F., Kühner, C., 2006. Beck Depressionsinventar - II. Harcourt, Frankfurt/Main.

- Hoyer, J., Markgraf, J., 2003. Angstdiagnostik. Grundlagen und Testverfahren. Springer, Berlin.

- Joels, M., Baram, Z., 2009. The neuro-symphony of stress. Nat. Rev. Neurosci. 10 (6), 459-466.

- Josephs, O., Henson, R.N., 1999. Event-related functional magnetic resonance imaging: modelling, inference and optimization. Philos. Trans. R. Soc. B 354, 1215 1228 .

- Katz, R.C.,Wilson, L., Frazer, N., 1994. Anxiety and its determinants in patients undergoing magnetic-resonance-imaging. J. Behav. Ther. Exp. Psychiatry 25 (2), 131134.

- Kinomura, S., Larsson, J., Gulyás, B., Roland, P.E., 1996. Activation by attention of the human reticular formation and thalamic intralaminar nuclei. Science 271, 512-517.

- Kirschbaum, C., Hellhammer, D.H., 1994. Salivary cortisol in psychoneuroendocrine research: recent developments and applications. Psychoneuroendocrinology 19 (4), 313-333.

- Kirschbaum, C., Pirke, K.M., Hellhammer, D.H., 1993. The 'Trier Social Stress Test' - a tool for investigating psychobiological stress responses in a laboratory setting. Neuropsychobiology 28 (1-2), 76-81.

- Kirschbaum, C., Bono, E.G., Rohleder, N., Gessner, C., Pirke, K.M., Salvador, A., Hellhammer, D.H., 1997. Effects of fasting and glucose load on free cortisol response to stress and nicotine. J. Clin. Endocrinol. Metab. 82 (4), 1101-1105.

- Kukolja, J., Schläpfner, T.E., Keysers, C., Klingmüller, D., Maier, W., Fink, G.R., Hurlemann, R., 2008a. Modeling a negative response bias in the human amygdala be noradrenergic-glucocorticoid interactions. J. Neurosci. 28 (48), 12868-12876.

- Kukolja, J., Thiel, C.M., Wolf, O.T., Fink, G.R., 2008b. Increased cortisol levels in cognitively challenging situations are beneficial in young but not older subjects. Psychopharmacology (Berl) 201 (2), 293-304.

- Laux, L., Glanzmann, P., Schaffner, P., Spielberger, C.D., 1981. State-TraitAngstinventar (STAI). Theoretische Grundlagen und Handanweisung. Beltz, Weinheim.

- $\quad$ Lupien, S.J., Maheu, F., Tu, M., Fiocco, A., Schramek, T.E., 2007. The effects of stress and stress hormones on human cognition: implications for the field of brain and cognition. Brain Cogn. 65 (3), 209-237. 
- Maldjian, J.A., Laurienti, P.J., Burdette, J.B., Kraft, R.A., 2003. An automated method for neuroanatomic and cytoarchitectonic atlas based interrogation of fMRI datasets. Neuroimage 19 (3), 1233-1239.

- McGaugh, J.L., Roozendaal, B., 2002. Role of adrenal stress hormones in forming lasting memories in the brain. Curr. Opin. Neurobiol. 12 (2), 205-210.

- McGlynn, F.D., Karg, R., Lawyer, S.R., 2003. Fear responses to mock magnetic resonance imaging among college students: toward a prototype experiment. J. Anxiety Disord. 17 (3), 335-347.

- McGlynn, F.D., Smitherman, T.A., Hammel, J.C., Lazarte, A.A., 2007. Component fears of claustrophobia associated with mock magnetic resonance imaging. J. Anxiety Disord. 21 (3), 367-380.

- McIsaac, H.K., Thordarson, D.S., Shafran, R., Rachman, S., Poole, G., 1998. Claustrophobia and the magnetic resonance imaging procedure. J. Behav. Med. 21 (3), 255-268.

- Melendez, J.C., McCrank, E., 1993. Anxiety-related reactions associated with magneticresonance- imaging examination. JAMA-J. Am. Med. Assoc. 270, 745-747.

- Morilak, D.A., Barrera, G., Echevarria, D.J., Garcia, A.S., Hernandez, A., Ma, S., Petre, C.O., 2005. Role of brain norepinephrine in the behavioral response to stress. Prog. Neuropsychopharmacol. Biol. Psychiatry 29 (8), 1214-1224.

- Nater, U.M., Rohleder, N., 2009. Salivary alpha-amylase as a non-invasive biomarker for the sympathetic nervous system: current state of research.

Psychoneuroendocrinology 34 (4), 486-496.

- Oei, N.Y., Everaerd, W.T., Elzinga, B.M., van Well, S., Bermond, B., 2006. Psychosocial stress impairs working memory at high loads: an association with cortisol levels and memory retrieval. Stress 9 (3), 133-141.

- Oei, N.Y., Tollenaar, M.S., Spinhoven, P., Elzinga, B.M., 2009. Hydrocortisone reduces emotional distracter interference in working memory.

Psychoneuroendocrinology 34 (9), 1284-1293.

- Radomsky, A.S., Rachman, S., Thordarson, D.S., McIsaac, H.K., Teachman, B.A., 2001. The claustrophobia questionnaires. J. Anxiety Disord. 15 (4), 287-297.

- Reul, J.M., de Kloet, E.R., 1986. Anatomical resolution of two types of corticosterone receptor sites in rat brain with in vitro autoradiography and computerized image analysis. J. Steroid Biochem. 24 (1), 269-272.

- Schneider, F., Gur, R.C., Gur, R.E., Muenz, L.R., 1994. Standardized mood induction with happy and sad facial expressions. Psychiatry Res. 51 (1), 19-31.

- Schoofs, D., Preuss, D., Wolf, O.T., 2008. Psychosocial stress induces working memory impairments in an n-back paradigm. Psychoneuroendocrinology 33 (5), 643653.

- Steyer, R., Schwenkmetzger, P., Notz, P., Eid, M., 1994. Testtheoretische Analysen des Mehrdimensionalen Befindlichkeitsfragebogens (MDBF). Diagnostica 40, 320328.

- $\quad$ Steyer, R., Schwenkmetzger, P., Notz, P., Eid, M., 1997. Mehrdimensionaler Befindlichkeitsfragebogen. Hogrefe, Göttingen.

- Tessner, K.D., Walker, E.F., Hochman, K., Hamann, S., 2006. Cortisol responses of healthy volunteers undergoing magnetic resonance imaging. Hum. Brain Mapp. 27 (11), 889-895.

- Valentino, R.J., Van Bockstaele, E., 2008. Convergent regulation of locus coeruleus activity as an adaptive response to stress. Eur. J. Pharmacol. 583 (2-3), 194-203. van Stegeren, A.H., Goekoop, R., Everaerd, W., Scheltens, P., Barkhof, F., Kuijer, J.P., 
- Rombouts, S.A., 2005. Noradrenaline mediates amygdala activation in men and women during encoding of emotional material. Neuroimage 24 (3), 898-909.

- $\quad$ van Stegeren, A., Rohleder, N., Everaerd, W., Wolf, O.T., 2006. Salivary alpha amylase as marker for adrenergic activity during stress: effect of betablockade. Psychoneuroendocrinology 31 (1), 137-141.

- $\quad$ van Stegeren, A.H., Roozendaal, B., Kindt, M., Wolf, O.T., Joels, M., 2010. Interacting noradrenergic and corticosteroid systems shift human brain activation patterns during encoding. Neurobiol. Learn. Mem. 93 (1), 56-65.

- Weerda, R., Muehlhan, M., Wolf, O.T., Thiel, C.M., 2010. Effects of acute psychosocial stress on working memory related brain activity in man. Hum. Brain Mapp. 31 (9), 1418-1429.

- Wolf, O.T., 2008. The influence of stress hormones on emotional memory: relevance for psychopathology. Acta Psychologica (Amst) 127 (3), 513-531.

- Wust, S., Wolf, J., Hellhammer, D.H., Federenko, I., Schommer, N., Kirschbaum, C., 2000. The cortisol awakening response - normal values and confounds. Noise Health 2 (7), 79-88. 